\title{
NECESSIDADE DE REABILITAÇÃO ORAL E ACESSO AOS SERVIÇOS ODONTOLÓGICOS DE IDOSOS INSTITUCIONALIZADOS NO MUNICÍPIO DE FEIRA DE SANTANA-BA
}

\section{ORAL REHABILITATION NEEDS AND ACCESS OF INSTITUTIONALIZED ELDERLY PEOPLE TO ORAL HEALTH SERVICES IN THE CITY OF FEIRA DE SANTANA-BA}

\author{
Nelia Medeiros Sampaio*, Mario Cezar Oliveira**, Adriana Lira Ortega***, Murilo Sampaio**** \\ Autor para correspondência: Mario Cezar Oliveira - mcezar11@@gmail.com \\ * Professora Assistente do Departamento de Saúde, Curso de Odontologia, Área de Prótese Dentária da \\ Universidade Estadual de Feira de Santana, Bahia \\ **Professor Adjunto do Departamento de Saúde, Curso de Odontologia, Área de Prótese Dentária da \\ Universidade Estadual de Feira de Santana, Bahia \\ ***Professora Adjunta do curso de Graduação e Pós-graduação do Instituto de Odontologia da Universidade \\ Cruzeiro do Sul - UNICSUL/SP \\ ****Professor Adjunto do Departamento de matemática da Universidade Federal de Sergipe
}

\section{R E S U M O}

\begin{abstract}
Descrever as condições sociodemográficas, necessidade de reabilitação oral e acesso aos serviços odontológicos de idosos institucionalizados no município de Feira de Santana, Bahia. Realizou-se um estudo transversal com 307 idosos, 80 residentes em Instituições de Longa Permanência para Idosos (ILPI) e 227 não-institucionalizados. Quanto à necessidade de reabilitação bucal, os idosos institucionalizados apresentaram maiores frequências de indivíduos que não usavam prótese dentária (68,35\%), quando usavam, 52,0\% delas foram consideradas inadequadas. Evidenciou-se o edentulismo como problema de saúde pública da pessoa idosa e que o grupo populacional formado por idosos institucionalizados demonstraram maiores necessidades de reabilitação bucal, bem como menor acesso a serviços odontológicos quando comparados ao grupo de idosos não institucionalizados na cidade de Feira de Santana-Ba.
\end{abstract}

Palavras-chave: Idoso, Epidemiologia, Saúde Bucal. 
Describe the sociodemographic conditions, needs for oral rehabilitation and access to dental care of institutionalized elderly in the city of Feira de Santana, Bahia. A cross-sectional study of 307 elderly people, 80 residents in long-stay institutions for the elderly (LSI) and 227 non-institutionalized. For the oral rehabilitation needs, the institutionalized had higher frequencies of individuals who did not use dental prothesis $(68.35 \%)$, when used $52.0 \%$ of them were considered inadequade. Edentulism was noticed up as a public health problem of the elderly and the group formed by institutionalized elderly population showed higher oral rehabilitation needs, as well as less access to dental services when compared with the group not institutionalized in Feira de Santana-Ba.

Key words: Aged, Epidemiology, Oral Health 


\section{INTRODUÇÃO}

A transição demográfica que vem ocorrendo no mundo, consequente da redução das taxas de fecundidade e das taxas de crescimento populacional se traduz em um elevado aumento da população idosa. No Brasil, segundo o Instituto Brasileiro de Geografia e Estatística (IBGE), o número de pessoas acima de 65 anos corresponde a $5 \%$ da população nacional e a expectativa é de que em 2050 o contingente populacional idoso poderá ser de 13,7 milhões de indivíduos'.

A irreversibilidade do envelhecimento populacional brasileiro deve ser encarada não só como uma conquista, mas como responsabilidade de toda a nossa sociedade ${ }^{2}$. É preciso conhecer as especificidades deste grupo populacional e assim planejar e subsidiar o desenvolvimento de políticas de saúde adequadas à realidade e necessidades da população idosas ${ }^{3,4}$.

As principais alterações bucais encontradas em indivíduos idosos, através dos estudos epidemiológicos são: a cárie dentária, a doença periodontal, abrasões, o câncer bucal e em destaque o edentulismo $0^{3,5}$.

Desta forma, são essenciais estudos epidemiológicos de boa qualidade e delineados de forma a contemplar tais necessidades. Considerando ainda, que as alterações bucais podem variar entre idosos não institucionalizados e institucionalizados ${ }^{3}$, esse estudo foi delineado com intuito de descrever as condições sócio-demográficas, necessidade de reabilitação oral e as condições de acesso aos serviços odontológicos de idosos no município de Feira de Santana, Bahia.

\section{MATERIAIS E MÉTODOS}

Através de um estudo epidemiológico de corte transversal, foi avaliado um grupo populacional composto por 307 idosos, sendo 80 residentes em Instituições de Longa Permanência para Idosos (ILPI) e 227 idosos não-institucionalizados. Foram considerados aptos para a pesquisa todos os moradores das ILPIs cadastrados na secretária de Desenvolvimento Social da Prefeitura de Feira de Santana e todos os idosos matriculados, que estivessem freqüentando as oficinas do programa Universidade Aberta a Terceira Idade (UATI) Universidade Estadual de Feira de Santana (UEFS) durante o semestre $2014^{2}$.

O treinamento e a calibração da equipe, composta por quatro examinadores, ocorreram em uma ILPI. A análise de concordância kappa, resultou em valores de 0,78 para o uso e 0,76 para a necessidade de próteses dentárias. Foi considerado também como critério de inclusão para os indivíduos no estudo a capacidade de responder as questões do instrumento de pesquisa. Para caracterizar o indivíduo como idoso, seguiu-se a recomendação da Organização Mundial da Saúde (OMS) considerando uma pessoa idosa, aquela com 60 anos de idade, para os países em desenvolvimento ${ }^{6}$.

Para coleta de dados foi utilizado um instrumento previamente elaborado e padronizado para registrar os dados de identificação do idoso (nome, sexo, idade), o perfil socioeconômico-demográfico, características do acesso a serviços odontológicos e também os resultados do exame clínico intrabucal que avaliou a necessidade de reabilitação bucal. Para a realização do exame clínico, utilizouse espátulas de madeira, luvas, gorros e máscaras descartáveis. Os exames intrabucais foram realizados sob iluminação natural nas sedes das ILPIs (idosos institucionalizados) e nas reuniões da UATI (idosos não institucionalizados).

A necessidade de reabilitação bucal foi mensurada a partir de informações sobre o edentulismo, exame clínico, uso e qualidade das próteses dentárias, tempo de uso e se houve acompanhamento após a instalação das mesmas. Para avaliar a qualidade das próteses dentárias removíveis (parcial ou total), foram considerados os seguintes critérios: retenção, estabilidade, reciprocidade, fixação, higiene, estética, dentes artificiais, baseados no Índice de Qualidade do Trabalho Protético (IQP) de Gil e Nakamae em 20007 que classifica a prótese em péssima, ruim, aceitável e adequada.

As informações foram armazenadas em um banco de dados e analisadas estatisticamente de forma 
descritiva e exploratória, utilizando o programa $R$ versão 2.9.0.

O estudo foi submetido à apreciação do Comitê de Ética em Pesquisa da Universidade Estadual de Feira de Santana - UEFS, e registrado sob o
Protocolo no 0005/2010, CAEE 0006.0.059.00010. Todos os indivíduos que aceitaram participar voluntariamente da pesquisa depois de esclarecidos sobre os propósitos do estudo assinaram um termo de consentimento livre e esclarecido.

\section{RESULTADOS}

A tabela 1 apresenta o universo do estudo, formado por 307 idosos distribuídos em 02 grupos populacionais distintos, um composto por 227 idosos não institucionalizados participante da UATI/UEFS, e outro por 80 idosos residentes nas quatro (04) ILPI do município, sendo três (03) filantrópicas e uma (01) particular.

Tabela 1. Distribuição da polução de idosos participantes do estudo, segundo a condição de institucionalização.

\begin{tabular}{cccccc}
\hline Grupo & Instituições & $\begin{array}{c}\text { Tipo de } \\
\text { instituições }\end{array}$ & Total & Excluídos & Participantes \\
\hline $\begin{array}{c}\text { Idosos não } \\
\text { institucionalizados }\end{array}$ & ---- & ----- & 458 & 231 & 227 \\
\hline \multirow{2}{*}{$\begin{array}{c}\text { Idosos } \\
\text { institucionalizados }\end{array}$} & Instituição I & Filantrópica & 37 & 07 & 30 \\
\cline { 2 - 6 } & Instituição II & Filantrópica & 20 & 06 & 14 \\
\cline { 2 - 6 } & Instituição III & Filantrópica & 63 & 39 & 12 \\
\hline
\end{tabular}

Na tabela 2 são apresentadas as características sociodemográficas dos idosos, notou-se uma maior freqüência do sexo feminino com renda mensal de um a três salários mínimos.

\begin{tabular}{|c|c|c|c|c|}
\hline \multirow[b]{2}{*}{ Variáveis } & \multicolumn{2}{|c|}{ Não-Institucionalizados(227) } & \multicolumn{2}{|c|}{ Institucionalizados (80) } \\
\hline & $\mathbf{N}$ & $\%$ & $\mathbf{N}$ & $\%$ \\
\hline \multicolumn{5}{|l|}{ Sexo (N 307) } \\
\hline Feminino & 208 & 91,6 & 53 & 66,2 \\
\hline Masculino & 19 & 08,4 & 27 & 33,8 \\
\hline \multicolumn{5}{|l|}{ Grupo éfnico (N 305) } \\
\hline Branco & 46 & 20,3 & 17 & 21,8 \\
\hline Preto & 43 & 18,9 & 11 & 14,1 \\
\hline Pardo & 138 & 60,8 & 50 & 64,1 \\
\hline \multicolumn{5}{|l|}{ Faixa Etária (N 303) } \\
\hline $60 a 70$ & 126 & 55,5 & 31 & 40,8 \\
\hline 71 a 80 & 82 & 36,1 & 26 & 34,2 \\
\hline 81 ou mais & 19 & 08,4 & 19 & 25,0 \\
\hline \multicolumn{5}{|l|}{ Estado Civil (N 305) } \\
\hline Solteiro & 29 & 12,9 & 38 & 47,5 \\
\hline Casado & 62 & 27,6 & 09 & 11,3 \\
\hline Divoc./Separ. & 28 & 12,4 & 08 & 10,0 \\
\hline Viúvo & 106 & 47,1 & 25 & 31,2 \\
\hline \multicolumn{5}{|l|}{ Escolaridade (N 304) } \\
\hline Analfabeto & 02 & 00,9 & 36 & 46,8 \\
\hline Antigo primário & 144 & 63,4 & 35 & 45,5 \\
\hline Antigo Ginásio & 67 & 29,5 & 04 & 05,2 \\
\hline Ensino superior & 14 & 06,7 & 02 & 02,6 \\
\hline \multicolumn{5}{|l|}{ Renda (N 255) } \\
\hline Abaixo de um salário mínimo & 1,07 & 03,4 & 01 & 02,0 \\
\hline De 01 a 03 salários mínimos & 164 & 80,4 & 48 & 94,1 \\
\hline Maior que 03 salários mínimos & 33 & 16,2 & 02 & 03,9 \\
\hline
\end{tabular}

A necessidade de reabilitação bucal é apresentada na tabela 3, observa-se que para os dois grupos populacionais a maior freqüência é de edêntulos parciais, sendo a prótese total bimaxilar foi o tipo de reabilitação bucal mais encontrada. Ainda na tabela 3 , notou-se que entre os idosos não institucionalizados houve uma maior freqüência daqueles que usavam prótese dentária $(81,78 \%)$, quando avaliou-se a qualidade 
destas próteses, 39,67\% foram consideradas aceitáveis. Já entre os idosos institucionalizados, as maiores freqüências são de indivíduos que não utilizavam prótese dentária $(68,35 \%)$, com $52,0 \%$ dessas próteses consideradas como ruins.

Tabela 3. Necessidade de reabilitação bucal entre os idosos participantes do estudo

\begin{tabular}{|c|c|c|c|c|}
\hline \multirow[b]{2}{*}{ Variável } & \multicolumn{2}{|c|}{ Não Institucionalizado (227) } & \multicolumn{2}{|c|}{ Institucionalixado (80) } \\
\hline & $\mathbf{N}$ & $\%$ & $\mathbf{N}$ & $\%$ \\
\hline \multicolumn{5}{|l|}{ Edentulismo } \\
\hline Total & 68 & 29,96 & 38 & 47,50 \\
\hline Parcial & 159 & 70,04 & 42 & 52,50 \\
\hline \multicolumn{5}{|l|}{ Usa prótese dentária } \\
\hline Sim & 184 & 81,78 & 25 & 31,65 \\
\hline Não & 41 & 18,22 & 54 & 68,35 \\
\hline \multicolumn{5}{|l|}{ Tipo de prótese } \\
\hline PT bimaxilar & 60 & 36,81 & 11 & 45,83 \\
\hline PT sup e PPR inf. & 39 & 25,15 & 04 & 16,67 \\
\hline PPR bimaxilar & 23 & 14,11 & 01 & 04,17 \\
\hline PT ou PPR em um dos arcos & 41 & 23,93 & 08 & 33,33 \\
\hline \multicolumn{5}{|l|}{ Qualidade PT e PPR } \\
\hline Boa & 51 & 27,72 & 05 & 20,00 \\
\hline Aceitável & 73 & 39,67 & 04 & 16,00 \\
\hline Ruim & 51 & 27,72 & 13 & 52,00 \\
\hline Péssima & 09 & 04,89 & 03 & 12,00 \\
\hline \multicolumn{5}{|l|}{ Controle da prótese } \\
\hline Não houve & 88 & 49,16 & 11 & 47,83 \\
\hline Houve & 85 & 47,49 & 09 & 39,13 \\
\hline Não soube responder & 06 & 03,35 & 03 & 13,04 \\
\hline \multicolumn{5}{|l|}{ Tempo de uso da prótese } \\
\hline Menos de 1 ano & 39 & 21,31 & 00 & 0,0 \\
\hline De 1 a 5 anos & 70 & 38,25 & 06 & 28,57 \\
\hline De 6 a 10 anos & 30 & 16,39 & 05 & 23,81 \\
\hline Mais de 10 anos & 44 & 24,05 & 10 & 47,62 \\
\hline \multicolumn{5}{|c|}{ Necessidade de troca ou uso de prótese dentária? } \\
\hline Sim & 136 & 59,91 & 37 & 46,25 \\
\hline Não & 91 & 40,09 & 36 & 45,00 \\
\hline Não soube responder & 00 & 0,0 & 07 & 08,75 \\
\hline
\end{tabular}

As características do acesso a serviços odontológicos entre os participantes estão apresentadas na tabela 4. Foram observadas diferenças quanto ao tempo e o motivo da última consulta ao cirurgião-dentista.

Tabela 4. Acesso aos serviços odontológicos entre os idosos participantes do estudo.

\begin{tabular}{|c|c|c|c|c|}
\hline \multirow[b]{2}{*}{ Veriável } & \multicolumn{2}{|c|}{ Não Instiłucionalixado (227) } & \multicolumn{2}{|c|}{ Instifueionalixado (80) } \\
\hline & $\mathbf{N}$ & $\%$ & $\mathbf{N}$ & $\%$ \\
\hline \multicolumn{5}{|l|}{ Quando foi a ultima consulta ao dentisła } \\
\hline Menos de 1 ano & 40 & 51,13 & 08 & 10,13 \\
\hline Um a dois anos & 58 & 32,95 & 07 & 08,86 \\
\hline Três anos ou mais & 67 & 38,87 & 52 & 65,82 \\
\hline Não soube responder & 11 & 06,25 & 12 & 15,19 \\
\hline \multicolumn{5}{|l|}{ Tipo de serviço } \\
\hline Serviço público & 12 & 05,29 & 22 & 27,50 \\
\hline Serviço privado & 160 & 70,49 & 32 & 40,00 \\
\hline Plano de Saúde & 30 & 13,21 & 03 & 03,75 \\
\hline Outros & 24 & 10,57 & 11 & 13,75 \\
\hline Não soube responder & 01 & 0,44 & 12 & 15,00 \\
\hline \multicolumn{5}{|l|}{ Motivo dea Consulter } \\
\hline Revisão & 55 & 24,23 & 05 & 06,25 \\
\hline Dor & 02 & 0,88 & 01 & 01,25 \\
\hline Extração & 26 & 11,45 & 42 & 52,50 \\
\hline Tratamento & 35 & 15,42 & 04 & 05,00 \\
\hline Prótese & 109 & 48,02 & 21 & 26,25 \\
\hline Não soube responder & 00 & 0,0 & 07 & 08,75 \\
\hline \multicolumn{5}{|l|}{ O que achou da ultima consulta } \\
\hline Muito Bom & 39 & 17,34 & 06 & 07,50 \\
\hline Bom & 131 & 58,22 & 42 & 52,50 \\
\hline Regular & 36 & 16,00 & 16 & 20,00 \\
\hline Muito ruim & 12 & 05,33 & 03 & 03,75 \\
\hline Ruim & 02 & 0,89 & 00 & 0,0 \\
\hline Não soube responde & 05 & 2,22 & 13 & 16,25 \\
\hline \multicolumn{5}{|l|}{ Grau de satisfação da condição bucal } \\
\hline Muito Satisfeito & 01 & 0,44 & 00 & 0,0 \\
\hline Satisfeito & 103 & 45,37 & 25 & 31,25 \\
\hline Nem satisfeito nem insatisfeito & 17 & 07,49 & 16 & 20,00 \\
\hline Insatisfeito & 105 & 46,25 & 30 & 37,50 \\
\hline Muito Insatisfeito & 01 & 0,44 & 01 & 01,25 \\
\hline Não soube responder & 00 & 0,0 & 08 & 10,00 \\
\hline
\end{tabular}




\section{DISCUSSÃO}

Os resultados do presente estudo são representativos para o grupo de idosos examinados, mas não podem ser generalizados para toda a população idosa do município de Feira de Santana. Possui as limitações de um estudo transversal e deve-se considerar a possibilidade de viés de resposta, pois às conclusões foram fundamentadas em informações auto-referidas, fato que pode afetar qualquer inquérito epidemiológico ${ }^{8,9}$.

O município de Feira de Santana, Bahia é contemplado com 04 (quatro) IPLI cadastradas na Secretaria Municipal de Desenvolvimento Social. Na ocasião da coleta dos dados foram encontrados 139 idosos residentes nestas instituições, dos quais 59 foram excluídos, ora porque possuíam idade inferior a 60 anos, ora por que sua saúde física e/ou mental não permitiu a realização do exame. De acordo com Gomes, Oliveira e Paiva, 20112 a proporção de idosos que vivem em IPLI depende de fatores culturais, grau de suporte familiar e disponibilidade de serviços alternativos.

As características sociodemográficas da população estudada refletem as atuais características de idosos em outras regiões do país, com predomínio do sexo feminino, de cor da pele parda, com baixa escolaridade e baixa renda ${ }^{2,10,11,12}$. Em alguns aspectos foi notada homogeneidade entre os dois grupos estudados e em outros fatores evidenciou-se algumas diferenças.

O predomínio de idosas no estudo é um fato esperado e pode ser explicado pelo número absoluto de mulheres ser superior ao de homens no Brasill. Em 2006, Camarano ${ }^{14}$ argumenta que em 1996, dos 12,4 milhões de idosos $54,4 \%$ eram do sexo feminino. Isso se deve à sua mais elevada taxa de crescimento relativamente à do segmento masculino. A maior longevidade da população feminina também explica esse diferencial na composição por sexo. Como conseqüência, quanto "mais velho" for o contingente estudado maior a proporção de mulheres neste. Este fato tem repercussões importantes nas demandas por políticas públicas

Quanto à renda, percebe-se no estudo uma clara homogeneidade, com predomínio de uma renda baixa, variando ao correspondente de 01 (um) a 02 (dois) salários mínimos. $O$ direito garantido na Constituição Federal, que assegura um salário mínimo mensal ao idoso com idade de 65 anos ou mais, através do Benefício de Prestação Continuada (BPC), é um fato que influencia nesta homogeniedade ${ }^{13}$.

As diferenças em relação ao estado civil entre os grupos é observada em outros estudos, sempre com predominância de idosos solteiros nas ILPIs $^{10}$. De acordo com Freitas \& Scheicherl, $2010^{14}$, as relações interpessoais do idoso e de sua família interferem no estilo e qualidade de vida destes, independente se institucionalizados ou não.

A criação de instituições filantrópicas no Brasil, destinadas a prestar cuidados a idosos sob a denominação de asilos, em sua origem, deu-se no século $X X$, e visava atender a velhice desamparada, que se configurava como uma população pobre e sem vínculos familiares ${ }^{15}$. Porém, as relações familiares em uma sociedade na qual a expectativa de vida está se expandindo podem suscitar situações inovadoras, sem que haja, o preparo das famílias para lidarem com essa realidade.

Os dados sobre analfabetismo revelam que este é acentuado entre os idosos'. O analfabetismo impede qualquer acesso à cultura escrita e a um conjunto de informações necessárias ao exercício da cidadania, sendo um dos mais graves indicadores de exclusão educacional e social. As estatísticas mostram também que a região Nordeste é a que apresenta os maiores índices de analfabetismo do país'.

Em relação ao grau de escolaridade, os resultados deste estudo confirmaram esses achados, e evidencia as diferenças entre os grupos populacionais. Entre os idosos não institucionalizados as maiores frequiências observadas foram dos que possuíam um grau maior de escolaridade, onde $27,3 \%$ havia concluído o ensino médio. Todavia, ao considerar que $22,5 \%$ desse grupo declararam possuir baixa escolaridade, pode-se aceitar o conceito de analfabetos funcionais para esses indivíduos. Ficou também evidente a situação de exclusão educacional entre os idosos institucionalizados onde $46,8 \%$ se declaram analfabetos. 
Ao verificar a necessidade de reabilitação bucal nestes grupos populacionais, confirmamos os achados encontrados em outros estudos realizados no Brasil ${ }^{10,16}$. Evidenciou-se uma alta prevalência do edentulismo, sendo os desdentados parciais os mais freqüentes nos dois grupos. Esses achados, além de refletir o efeito da prática odontológica mutiladora ao longo da vida desses indivíduos, também desnuda a ineficiência das formas de planejamento de programas que apresentam características excludentes de acesso ${ }^{3,12}$.

O edentulismo total foi mais observado no grupo de idosos institucionalizados, sendo $47,50 \%$ e de desdentados parciais 52,50\%. Segundo Barbato \& Peres, $2015^{18}$, a exclusão das instituições para idosos nas políticas públicas torna-se um fator representativo das piores condições bucais nesse grupo populacional.

Sobre essa afirmação, quando se observa dados referentes ao uso de prótese dentária, no grupo dos não institucionalizados foi notado que $81,78 \%$ utilizam próteses dentárias, que ao passar por uma avaliação de qualidade a maioria destas foram consideradas como aceitáveis $(39,67 \%)$ e que estavam em um período de uso de 01 (um) a 05 (cinco) anos $(38,25 \%)$. Entretanto, no grupo populacional de idosos institucionalizados $68,35 \%$ não utilizavam prótese dentárias, a maioria das que estavam em uso foram consideradas ruins $(52,0 \%)$ e $47,62 \%$ estavam em uso por mais de 10 (dez) anos. Porém quando questionado sobre o acompanhamento após a instalação das próteses dentárias, ressaltase que nos dois grupos a maior frequiência foi do não acompanhamento após a instalação.

Giordani et al. em $2011^{19}$ ressaltaram a importância da reabilitação protética no amplo contexto da saúde para indivíduos idosos, como também para a sua integração social. Consideram que a perda da dentição tem sérios impactos negativos sobre a mastigação, digestão, gustação, pronúncia e aspecto estético.

A falta de assistência odontológica, posterior à colocação da prótese, é um dos fatores que justifica os elevados percentuais de necessidade de reparo ou substituição, assim como a alta prevalência de lesões associadas às mesmas ${ }^{20}$. O estudo revela que os idosos institucionalizados tiveram na sua maioria $(65,82 \%)$ a última consulta odontológica há três anos ou mais, enquanto que $51,13 \%$ dos não institucionalizados foram a menos de um ano ao cirurgião-dentista.

Quando questionados sobre a necessidade de troca e/ou utilização de prótese dentária, a maioria nos dois grupos considerou a necessidade como positiva, demonstrando o interesse e necessidade de reabilitação bucal, e evidenciando as limitações de acesso a esses serviços. Nos dois grupos, apesar da baixa renda, o tipo de serviço a que teve acesso na última consulta ao cirurgião-dentista foi na sua maioria o serviço privado.

O motivo que levou o idoso a procurar assistência odontológica também demonstra diferenças sociais importantes, para os idosos não institucionalizados - motivo mais relatado foi a confecção de prótese dentária, enquanto que os institucionalizados foram em busca de exodontias. Piuvezam \& Lima em $2013^{21}$ argumentaram que, para idosos há uma cobertura insuficiente de atenção em saúde bucal, e que em países em desenvolvimento, ocorre o agravante da predominância de serviços focados no alívio da dor ou atendimento de emergência.

Para Mello, Erdmann e Brondani em 200722, essas diferenças entre os grupos populacionais, se justificam pelas ILPI ficarem à margem, excluídas de políticas públicas e, principalmente, de saúde bucal, $\circ$ idoso institucionalizado encontra-se adicionalmente excluído do acesso à atenção e assistência odontológicas públicas. O Estado Brasileiro, pela via dos serviços públicos de saúde, age passivamente, e assim se revela omisso no essencial, ao não reconhecer e atender às demandas presentes nas ILP para idosos. Uma esperança de mudanças deste quadro se deve à implantação da Política Nacional de Saúde Bucal ${ }^{23}$, criada com intuito para aumentar o acesso aos níveis secundários e terciários de atenção, visto que no setor odontológico, ao longo dos anos a expansão da rede assistencial de atenção nestes níveis não acompanhou o crescimento da oferta de serviços da atenção primária.

Ao questionamento sobre $\circ$ que achou da ultima consulta odontológica, os dados revelam passividade nos dois grupos, pois consideraram na sua maioria de boa qualidade. Porém ao serem 
indagados sobre o grau de satisfação da própria condição bucal foi mais freqüente a insatisfação. De acordo com Usual, Araujo, Diniz e Drumond em $2006^{24}$, a satisfação humana é um fator rodeado de complexidades que envolvem conceitos, valores, expectativas, experiências passadas e estilos de vida.

\section{CONCLUSÃO}

Ao caracterizar o grupo populacional de idosos, foi observado uma maior freqüência do sexo feminino, de cor da pele parda e baixa renda. Entre os institucionalizados e não institucionalizados houve diferenças marcantes quanto ao estado civil e a escolaridade.

O edentulismo ficou evidenciado como um problema de saúde pública do indivíduo idoso. Os institucionalizados apresentaram maior necessidade de reabilitação bucal, bem como menor acesso a serviços odontológicos quando comparados ao grupo dos não institucionalizados, evidenciando a necessidade da criação e implantação de políticas e ações de reabilitação bucal para idosos no município de Feira de Santana-Ba.

\section{REFERÊNCIAS}

1. Brasil. Instituto Brasileiro de Geografia e Estatística. Censo Populacional 2010. Brasília. DF. 2012

\section{Gomes MJ, Oliveira ERA, Paiva KM.} Institucionalização e qualidade de vida de idosos da região metropolitana de Vitória/ES. Esc Anna Nery. 2011 ; 15(3):518-23. doi: 10.1590/S141481452011000300011

3. Pucca Junior GA, Costa JFR, Chagas LD, Sivestre RM. Oral health policies in Brazil. Braz Oral Res. 2009;23:9-16. doi: 10.1590/S180683242009000500003

4. Roncalli AG, Tsakos G, Sheiham A, Souza GC, Watt RG. Social determinants of dental treatment needs in
Brazilian adults. BMC Public Health. 2014;14:1097. doi: $10.1590 /$ S1806-83242009000500003

5. Brasil. Ministério da Saúde. Secretaria de Atenção à Saúde. Secretaria de Vigilância em Saúde. Departamento de Atenção Básica. Projeto SB Brasil. Pesquisa Nacional de Saúde Bucal - Resultados Principais 2010. Brasília. DF. 2011

6. World Health Organization. Commission on Social Determinants of Health [internet]. [Acesso em 14 Set 2015]. 2015. [Disponível em: http://www. who.int/social_determinants/thecommission/finalreport/ key_concepts/en/]

7. Gil C, Nakamae AEM. Índice de qualidade do trabalho protético (IQP): um estudo metodológico. RPG Rev Pos-grad. 2000;7(1):38-46

8. Marmot $M$. The health gap: the challenge of an unequal world. Lancet. 2015;386:2442-44. doi: $10.1016 / \mathrm{s} 0140-6736(15) 00150-6$

9. Pereira MG. Epidemiologia: teoria e prática. 10 ed. Rio de Janeiro: Guanabara Koogan; 2006

10. Ferreira RC, Magalhães CS, Rocha ES, Schwambach CW, Moreira AN. Saúde bucal de idosos residentes em instituições de longa permanência de Belo Horizonte, Minas Gerais, Brasil. Cad. Saúde Pública. 2009;25(1 1):2375-2385. doi: 10.1590/S0102$311 \times 2009001100008$

11. Harford J. Population ageing and dental care. Community Dent Oral Epidemiol. 2009;37:97-103. doi: $10.1111 /$ j.1600-0528.2008.00441.x

12. Brasil. Instituto Brasileiro de Geografia e Estatística. PNAD 2006: síntese de indicadores sociais. Brasília. DF. 2006

13. Areosa SVC, Areosa AL. Envelhecimento e dependência: desafios a serem enfrentados. Revista Textos \& Contextos. 2008; $7(1): 138-50$

14. Freitas, MAV, Scheicher ME. Qualidade de vida de Idosos Institucionalizados. Revista Brasileira de Geriatria e Gerontologia. 2010;13(3):1 14-119. doi: 10.1590/ S1809-98232010000300006

15. Alcântara $A O$. Velhos institucionalizados e família: entre abafos e desabafos. São Paulo: Alínea; 2004

16. Martins AMEB, Barreto SM, Pordeus IA. Utilization of dental services among the elderly in Brazil. Pan Am 
17. De Visschere LM, Grooten L, Theuniers G, et al. Oral hygiene of elderly people in longterm care institutions - a cross sectional study. Gerodontology. 2006;23(4):195-204. doi: $10.1111 /$ i.17412358.2006.00139.x

18. Barbato PR, Peres KG. Contextual socioeconomic determinants of tooth loss in adults and elderly: a systematic review. Rev Bras Epidemiol. 2015;18(2):35771. doi: 10.1590/1980-5497201500020006

19. Giordani JMA, de Slavutzky SMB, Koltermann AP, Pattussi MP. Inequalities in prosthetic rehabilitation among elderly people: the importance of context. Community Dent Oral Epidemiol. 2011 ;39(3):230-8. doi: $10.1111 /$ i.1600-0528.2010.00587.x

20. Mariño R, Giacaman RA. Factors related to unmet oral health needs in older adults living in Chile. Arch Gerontol Geriatr. 2014;58(3):454-9. doi: 10.1016/i. archger.2014.01.003

21. Piuvezam G, Lima KC. Factors associated with missing teeth in the Brazilian elderly institutionalised population. Gerodontology. 2013; 30:141-9. doi: $10.1111 /$ j.1741-2358.2012.00655.x

22. Mello ALSF, Erdmann ALE, Brondani M. Oral health care in long-term care facilities for elderly people in southern Brazil: a conceptual framework. Gerodontology. 2010;27:41-46. doi: $10.1111 /$ j.1741 2358.2009.00280.x

23. Brasil. Ministério da Saúde. Desenvolvimento de Sistemas e Serviços de Saúde - A política nacional de saúde bucal do Brasil: registro de uma conquista histórica. Brasília. DF. 2006

24. Usual AB, Araujo AA, Diniz FVM, Drumond MM. Necessidade sentida e observada: suas influencias na satisfação de pacientes e profissionais. Arq Odontol. 2006;42(1):65-80 